\title{
Modelagem Inteligente Nebulosa Aplicada à Hidroponia via Sistema Embarcado
}

\author{
Kayon V. L. Lopes* Danúbia S. Pires* Orlando D. R. Filho* \\ Ginalber L. O. Serra* \\ * Departamento de Eletroeletrônica, Instituto Federal de Educação, \\ Ciência e Tecnologia do Maranhão (e-mail: \\ kayonvinicius222@hotmail.com; danubiapires@ifma.edu.br; \\ orlando.rocha@ifma.edu.br; ginalber@ifma.edu.br)
}

\begin{abstract}
This project aims to use the fuzzy Logic for automation and monitoring of the variables involved in the development of vegetables in hydroponic systems that require large human intervention. Hydroponics is an alternative protected cultivation technique in which the soil is replaced by an aqueous solution containing only the mineral elements essential to the plants and presents several advantages in relation to soil cultivation, such as: water economy, precocity in production, and minimal use of pesticides. This paper proposes the creation of a system capable of controlling the production and monitoring of vegetables in greenhouses, collecting and controlling essential variables such as temperature, humidity, luminosity, among others, using fuzzy logic as a modeling tool.

Resumo: Este projeto visa a utilização da Lógica Nebulosa para automatização e monitoramento das variáveis envolvidas no desenvolvimento de hortaliças em sistemas hidropônicos que exigem grande intervenção humana. A hidroponia é uma técnica alternativa de cultivo protegido, na qual o solo é substituído por uma solução aquosa contendo apenas os elementos minerais essenciais aos vegetais, e apresenta diversas vantagens em relação ao cultivo em solo, como: economia de água, precocidade na produção, e mínimo uso de defensivos agrícolas. Neste artigo propõe-se a criação de um sistema capaz de controlar a produção e o monitoramento de hortaliças em estufas para cultivo, coletando e controlando variáveis essenciais como temperatura, umidade, luminosidade, entre outras, utilizando-se da lógica nebulosa como ferramenta de modelagem.
\end{abstract}

Keywords: Hydroponics; Intelligent System; Fuzzy Control; Embedded Systems.

Palavras-chaves: Hidroponia; Sistema Inteligente; Controle Nebuloso; Sistemas Embarcados.

\section{INTRODUÇÃO}

A hidroponia é relativamente antiga, no entanto, só ganhou popularidade a partir de 1930 , com a criação da nomenclatura pelo Dr. W. F. Gericke, da universidade da Califórnia. Consiste em uma técnica para o cultivo de plantas, sem solo, em meio hídrico, onde as raízes recebem uma solução nutritiva balanceada que contém água e todos os nutrientes essenciais ao desenvolvimento da planta, podendo reduzir em até $70 \%$ a quantidade de água utilizada (dos Santos et al., 2013), garantindo economia, precocidade, e higiene do produto.

Atualmente é mais difundida em países como a Holanda, Alemanha, Itália, Japão, Estados Unidos, México e áreas vizinhas da América Central. No Brasil, entretanto, esta técnica ainda não é muito difundida, sendo mais utilizada perto dos grandes centros urbanos onde as terras férteis são mais escassas e caras (Loschiavo, 2015).

Apesar de ser ainda incipiente no país, a tendência é que haja um aumento significativo do uso dessa técnica

\footnotetext{
* Reconhecimento do suporte financeiro deve vir nesta nota de rodapé.
}

em diversas localidades onde o clima instável prejudica a produção agrícola.

Técnicas como esta ganharam muita importância ao longo dos anos, e estão sendo gradualmente empregadas para a agricultura comercial (JSM and Sridevi, 2014). Um dos sistemas mais utilizados é o NFT - Sistema de Fluxo Laminar de Nutrientes - onde as plantas crescem tendo seu sistema radicular dentro de um canal ou canaleta onde circula uma solução nutritiva. Este sistema é composto basicamente por um tanque de solução nutritiva, um sistema de bombeamento dos canais de cultivo e um sistema de retorno ao tanque. A solução nutritiva é bombeada aos canais e escoa por gravidade formando uma fina lâmina de solução que irriga as raízes (de Abreu et al., 2015). O sistema NFT surgiu na Inglaterra em 1965 e é hoje o principal método de hidroponia utilizado no Brasil (Hidroponia, 2013).

O sistema MIMO inteligente nebuloso aplicado ao cultivo hidropônico apresentado neste artigo tem como objetivo a redução de custos e de erros no controle da produção, uma vez que o controle correto dos processos em um 
sistema de cultivo hidropônico influencia diretamente na produtividade, qualidade e precocidade do produto.

\section{JUSTIFICATIVA}

O método de cultivo convencional apresenta várias desvantagens quando se trata de clima, uma vez que mudanças de temperatura, umidade, diminuição de incidência solar e outros fatores podem comprometer seriamente a safra causando prejuízo (de Abreu et al., 2015).

Neste sentido, a automação é extremamente necessária para minimizar os prejuízos, através do controle de variáveis tais como a temperatura, luminosidade, umidade do ar, alcalinidade da água, entre outras, em abrigos de cultivo. Além de minimizar o risco de perda de safra, a automação possibilita uma maior qualidade dos produtos por proporcionar condições ideais para produção, e permite autonomia no cultivo, possibilitando que a mão-de-obra humana seja direcionada a atividades que exigem maior dedicação.

Existem várias técnicas de cultivo, entretanto, alguns métodos alternativos se mostram mais eficientes em alguns aspectos em relação ao plantio convencional em solo. Um deles é o cultivo em ambientes isolados, como estufas, onde há uma menor possibilidade do surgimento de pragas, fungos, entre outros(de Abreu et al., 2015). Dentro dessas estufas são instalados sistemas de cultivo de acordo com a técnica desejada. Neste artigo, o sistema de automação será feito com objetivo de auxiliar a técnica hidropônica NFT (Fluxo Laminar de Nutrientes), já que esta, aliada à automação, possui muitas vantagens em relação ao plantio tradicional em solo, uma vez que um controle correto dos processos em abrigos de cultivo proporciona uma maior produtividade e melhor qualidade do produto (Alberoni, 1998).

Atualmente existem alguns trabalhos de automação hidropônica visando melhoria de controle em estufas, como em Goldstein (2018), Fonseca and Schmitz (2017), Azevedo Junior (2016), Matsura et al. (2015), Santos e Teruel (2010). No entanto, observa-se que esses trabalhos são baseados em lógica convencional (lógica booleana). Embora sejam eficientes em alguns casos, esses sistemas não permitem controlar a intensidade da iluminação ou ventilação fornecida por conta da sua natureza crisp. Assim, para o caso da iluminação e ventilação em estufas de cultivo, por exemplo, os estados possíveis seriam: ligado ou desligado. Neste contexto, a lógica nebulosa pode representar uma ferramenta eficiente na tomada de decisões, uma vez que utiliza raciocínio e linguagem natural, a fim de representar valores, os quais são expressos linguisticamente (Zanette et al., 2013), o que permite representar meios-termos em variáveis como, por exemplo, temperatura, que pode ser definida como alta, morna, média, baixa, entre outros, possibilitando o tratamento de incertezas, dando robustez ao sistema proposto, e proporcionando economia de energia ao permitir o controle de intensidade na iluminação e ventilação.

Com a utilização deste sistema, procura-se diminuir a intervenção humana na coleta de dados e no manejo do cultivo hidropônico, reduzindo os trabalhos repetitivos e os erros que normalmente acompanham estas atividades.

\section{METODOLOGIA}

O sistema inteligente proposto neste artigo tem como base um modelo nebuloso desenvolvido utilizando o método de inferência de Mamdani. Neste método, a $i$-ésima regra do modelo é dada por:

$$
\begin{gathered}
R^{(i)}: \mathrm{SE} x_{1} \text { É } A_{1}^{j *} \mathrm{E} \ldots \mathrm{E} x_{n} \text { É } A_{n}^{j *} \\
\text { ENTÃO } y_{1} \mathrm{E} B_{1}^{m *} \mathrm{E} \ldots \mathrm{E} y_{n} \mathrm{E} B_{n}^{m *}
\end{gathered}
$$

onde $x_{1, \ldots, n}$ corresponde às variáveis linguísticas do antecedente das regras nebulosas; $A_{1, \ldots, n}^{j *}$ correspondem às partições nebulosas das variáveis linguísticas; $y_{1, \ldots, n}$ são as saídas do modelo Mamdani, representadas pelas partições nebulosas $B_{1, \ldots, n}^{m *}$.

As entradas $x_{1, \ldots, n}$ do sistema foram definidas de acordo com a influência sobre o microclima dentro das estufas de cultivo. Em Rebouças et al. (2014), o controle e o monitoramento de variáveis meteorológicas, como temperatura e umidade, dentro de estufas hidropônicas é de suma importância, dado que as variações destas variáveis influenciam diretamente no desenvolvimento das culturas por meio de efeitos na transpiração e na fotossíntese, podendo interferir de maneira benéfica ou maléfica no desenvolvimento da planta. Além dos fatores supracitados, é importante considerar o efeito do horário e da incidência de radiação solar. Ainda em Rebouças et al. (2014), as maiores variações de temperatura ocorrem entre as 12 e 16 horas, intervalo em que a temperatura do ar está mais elevada. Essas variações na temperatura, por sua vez, influenciam a umidade relativa dentro das estufas de cultivo que, durante o período diurno, diminui com grande intensidade e, durante a noite, aumenta rapidamente. Assim, devido à importância das variáveis meteorológicas e do horário, bem como a incidência solar, as variáveis definidas como as entradas $x_{i}$ do sistema nebuloso proposto foram temperatura, umidade, período do dia e luminosidade.

As saídas $y_{i}$ escolhidas foram as variáveis iluminação e ventilação interna. A iluminação representa um fator de muita importância nas estufas, uma vez que nem sempre a exposição direta à luz solar é possível; a ventilação interna, por sua vez, pode proporcionar uma redução considerável na temperatura e umidade no interior da estufa. $\mathrm{O}$ valor de saída da variável iluminação é destinada a um módulo de LEDs por meio de Modulação da Largura de Pulso (PWM, do inglês, Pulse Width Modulation) (Hirzel, 2016) para controle da intensidade luminosa. A função deste módulo é proporcionar uma compensação da luz solar por meio de LEDs RGB com comprimento de onda entre $630 \mathrm{~nm}$ e $460 \mathrm{~nm}$, a fim de criar uma iluminação que possa suprir a necessidade de luz solar para garantir uma maior atividade fotossintética e, consequentemente, o pleno desenvolvimento das hortaliças cultivadas (Almeida, 2014). Alguns estudos, como em Jackson (2009), mostram técnicas que consistem no aumento do período de exposição solar, chamado fotoperíodo, visando um aumento de precocidade na produção das safras. Baseado nestes estudos, aplicou-se o acionamento da iluminação artificial no período das $18 \mathrm{~h}$ às $21 \mathrm{~h}$. Este intervalo foi definido como um conjuntos nebulosos da variável de entrada período, conforme o descrito na Tabela 1. A segunda variável 
de saída, ventilação, é destinada ao pino de controle de velocidade de duas ventoinhas para ajuste da ventilação por meio da exaustão e da entrada de ar no abrigo de cultivo.

Embora o sistema proposto possa subdividido em dois sistemas nebulosos independentes responsáveis pela iluminação e ventilação, devido à ausência de relação direta entre estas duas variáveis, optou-se por manter um único sistema, e, consequentemente, uma única base de regras, com o objetivo de evitar o aumento do custo computacional e tempo de resposta devido às instanciações dos dois sistemas diferentes.

A partir de um módulo de sensoriamento gerenciado por uma placa Arduino Mega, foi realizada a coleta de dados. Neste módulo, a aquisição dos valores das respectivas variáveis foi realizada por meio de sensores específicos para a plataforma Arduino. Utilizaram-se sensores DHT11 e DHT22 para obtenção dos dados de temperatura e umidade; um sensor LDR para medição dos valores de luminosidade e um módulo RTC DS1307 para obtenção do horário, conforme a Figura 1. A partir da análise de cada sensor foi definido o universo de discurso (UD) de cada entrada $x_{i}$ do sistema nebuloso.

Na etapa subsequente à coleta dos dados, tem-se a inserção destes no controlador nebuloso. Para isso, realizase a etapa de fuzzificação das variáveis $x_{i}$. Os valores obtidos durante a coleta dos dados são mapeados para valores no intervalo de 0 a 1 , dado por:

$$
\mu_{A}(x) \rightarrow[0,1]
$$

onde $\mu_{A}(x)$ é o grau de pertinência de $x$ ao conjunto $A$.

A fuzzyficação avalia o grau de pertinência da entrada numérica, $x_{i}$, com ativação das regras para composição dos conjuntos nebulosos de saída, os quais serão defuzzyficados gerando um resultado numérico para a saída $y_{i}$ (Cavalcanti et al., 2012).

As partições nebulosas $A_{1, \ldots, n}^{j *}$ foram definidas para cada variável $x_{i}$, e os conjuntos foram modelados utilizando funções de pertinência do tipo triangular considerando o suporte dos conjuntos nebulosos especificados nas Tabelas $1,2,3$, e 4 para as variáveis período, temperatura, umidade e luminosidade, respectivamente.

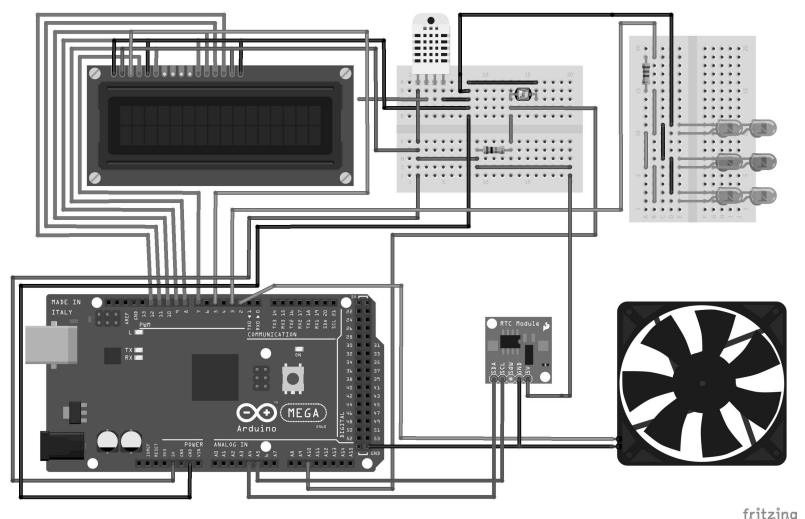

Figura 1. Modelo esquemático do sistema proposto.

Na Tabela 4, o UD da luminosidade é a faixa de valores compreendidos entre 0 a 1023. Este intervalo é
Tabela 1. Conjuntos nebulosos para a variável Período.

\begin{tabular}{|r|r|}
\multicolumn{2}{c}{ Período (Horas do dia) } \\
\hline Conjunto & Univ. de discurso \\
\hline Madrugada & $0<\mathrm{x}<5$ \\
\hline Manhã & $4<\mathrm{x}<13$ \\
\hline Tarde & $12<\mathrm{x}<19$ \\
\hline Sobretarde & $18<\mathrm{x}<21$ \\
\hline Noite & $20<\mathrm{x}<24$ \\
\hline
\end{tabular}

Tabela 2. Conjuntos nebulosos para a variável Temperatura.

\begin{tabular}{|} 
Temperatura $\left({ }^{\circ} \mathrm{C}\right)$ \\
\begin{tabular}{|r|r|}
\hline Conjunto & Univ. de discurso \\
\hline Baixa & $\mathrm{x}<25$ \\
\hline Média & $20<\mathrm{x}<37$ \\
\hline Alta & $\mathrm{x}>35$ \\
\hline
\end{tabular}
\end{tabular}

Tabela 3. Conjuntos nebulosos para a variável Umidade.

Umidade Relativa (\%)

\begin{tabular}{|r|r|}
\hline Conjunto & Univ. de discurso \\
\hline Baixa & $0<\mathrm{x}<60$ \\
\hline Ideal & $50<\mathrm{x}<80$ \\
\hline Alta & $70<\mathrm{x}<100$ \\
\hline
\end{tabular}

Tabela 4. Conjuntos nebulosos para a variável Luminosidade.

\begin{tabular}{|r|r|}
\multicolumn{2}{|c}{ Luminosidade } \\
\hline Conjunto & Univ. de discurso \\
\hline Baixa & $0<\mathrm{x}<580$ \\
\hline Média & $550<\mathrm{x}<700$ \\
\hline Alta & $680<\mathrm{x}<1023$ \\
\hline
\end{tabular}

resultado do mapeamento dos valores lidos pelo Arduino, a partir da tensão detectada no pino do sensor LDR (Lima, 2017). Esta tensão pode variar de 0 a $5 \mathrm{~V}$ e, por meio de um conversor analógico-digital de 10 bits de resolução, é mapeado para um faixa de 0 a 1023 unidades, onde cada uma corresponde a $4,9 \mathrm{mV}$ de tensão (Arduino, 2017). Por se tratar de um resistor dependente de luz, a relação entre o valor lido pelo Arduino e a incidência de luz é inversamente proporcional. Quanto maior a luminosidade no ambiente, menor será o valor lido.

As partições nebulosas $B_{1, \ldots, n}^{m *}$ foram definidas para cada variável de saída $y_{i}$, utilizando funções de pertinência triangulares considerando os UDs especificados nas Tabelas 5 e 6 .

Tabela 5. Conjuntos nebulosos para a variável de saída Ventilação.

\begin{tabular}{|r|r|}
\multicolumn{2}{|c}{ Ventilação } \\
\hline Conjunto & Univ. de discurso \\
\hline Desligada & $0<\mathrm{x}<95$ \\
\hline Média & $73<\mathrm{x}<179$ \\
\hline Alta & $160<\mathrm{x}<255$ \\
\hline
\end{tabular}

Tabela 6. Conjuntos nebulosos para a variável de saída Iluminação.

Iluminação

\begin{tabular}{|r|r|}
\multicolumn{1}{l}{ Iluminação } \\
\begin{tabular}{|r|r|}
\hline Conjunto & Univ. de discurso \\
\hline Desligada & $0<\mathrm{x}<361$ \\
\hline Média & $321<\mathrm{x}<702$ \\
\hline Alta & $662<\mathrm{x}<1023$ \\
\hline
\end{tabular}
\end{tabular}


Nas Figuras 2, 3, 4 e 5 são observadas as funções de pertinência das variáveis de entrada consideradas no sistema. Nas Figuras 6 e 7 são mostradas as funções de pertinência das variáveis de saída.

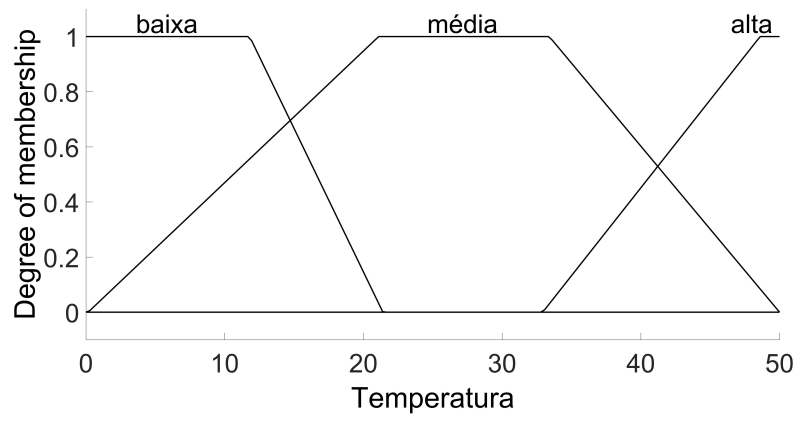

Figura 2. Funções de pertinência da variável Temperatura.

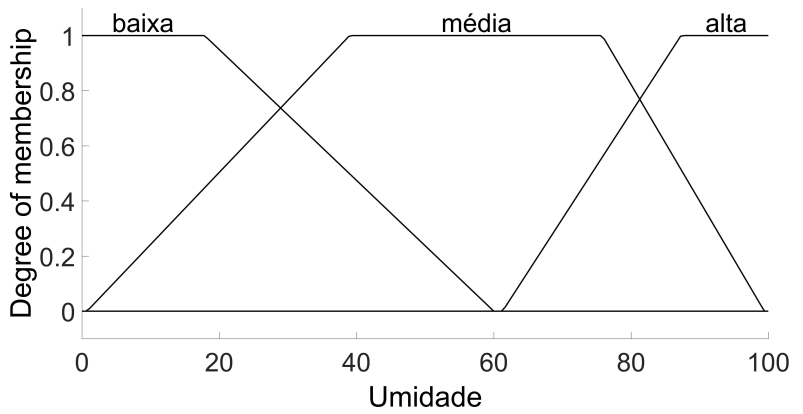

Figura 3. Funções de pertinência da variável Umidade.

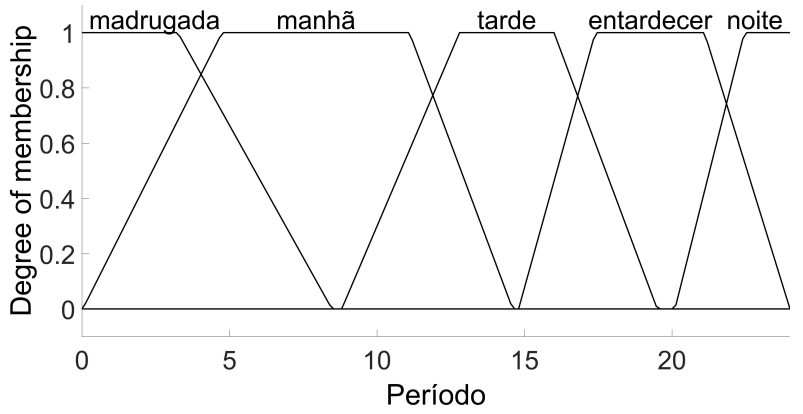

Figura 4. Funções de pertinência da variável Período.

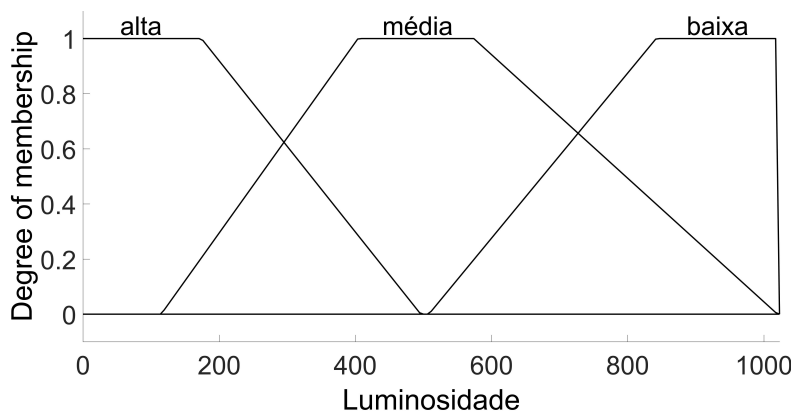

Figura 5. Funções de pertinência da variável Luminosidade.

Após a coleta dos dados e a fuzzificação dos valores de cada variável, responsável por realizar a conversão dos valores escalares para valores nebulosos correspondentes, inicia-se a etapa de processamento das regras existentes

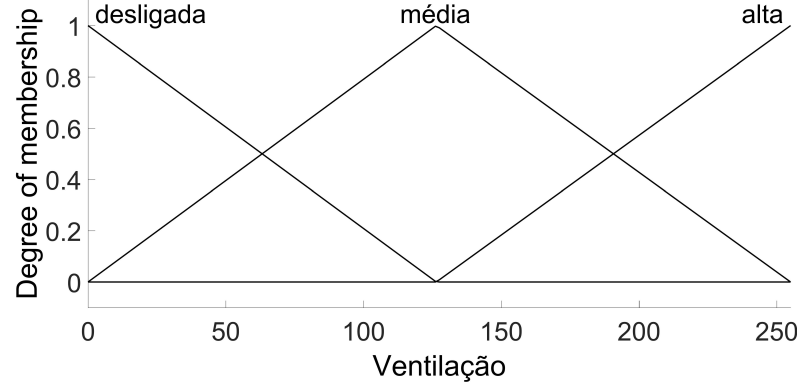

Figura 6. Funções de pertinência da variável Ventilação.

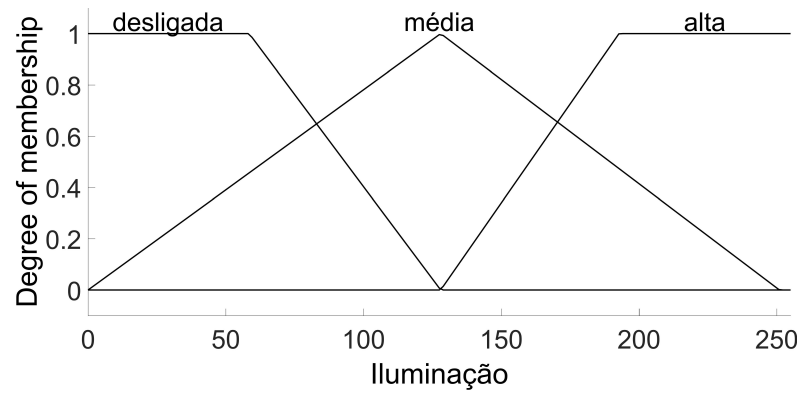

Figura 7. Funções de pertinência da variável iluminação.

na base de regras: a máquina de inferência nebulosa realiza as operações entre os conjuntos $A_{1, \ldots, n}^{j *}$, para obtenção dos conjuntos nebulosos $B_{1 \ldots \ldots n}^{m *}$. As regras possuem estrutura conforme Equação 1. Algumas das regras utilizadas no sistema proposto são observadas na Tabela 7 .

Tabela 7. Base de regras do sistema proposto

\begin{tabular}{|c|c|}
\hline & Regras Utilizadas \\
\hline $1^{\circ}$ & $\begin{array}{c}\text { Se Temperatura é Alta e Umidade é Alta } \\
\text { então Ventilação é Alta }\end{array}$ \\
\hline 2 & $\begin{array}{c}\text { Se Temperatura é Alta e Umidade é Média então } \\
\text { Ventilação é Alta }\end{array}$ \\
\hline 3 & $\begin{array}{c}\text { Se Temperatura é Alta e Umidade é baixa então } \\
\text { Ventilação é Alta }\end{array}$ \\
\hline$\vdots$ & $\vdots$ \\
\hline 11 & $\begin{array}{c}\text { Se Temperatura é baixa e Umidade é Alta então } \\
\text { Ventilação é Média }\end{array}$ \\
\hline 12 & $\begin{array}{c}\text { Se Luminosidade é baixa e Horário é Manhã então } \\
\text { Iluminação é Média }\end{array}$ \\
\hline 13 & $\begin{array}{c}\text { Se Luminosidade é Alta e Horário é Tarde então } \\
\text { Iluminação é Desligada }\end{array}$ \\
\hline$\vdots$ & Se Período é Noite então Iluminação é Desligada \\
\hline 27 & \begin{tabular}{c} 
Se Período é Madrugada então Iluminação é Desligada \\
\hline
\end{tabular} \\
\hline
\end{tabular}

Por fim, os conjuntos nebulosos obtidos pela inferência precisam ser convertidos para uma saída numérica, possibilitando o acionamento das saídas pela placa Arduino. Neste ponto é necessário realizar a defuzzificação da saída obtida na etapa de inferência. Para isto, foi utilizado o método do centróide que consiste no cálculo, para um dado conjunto nebuloso proveniente do processo de inferência, da abcissa do ponto de centro de massa (Rezende, 2003), dado conforme segue: 


$$
y *=\frac{\sum_{m=1}^{N_{y}} y_{m} \mu_{0}\left(y_{m}\right)}{\sum_{m=1}^{N_{y}} \mu_{0}\left(y_{m}\right)}
$$

onde $y_{m}$ é o valor central da regra $m$ e $N_{y}$ é o número de regras do sistema nebuloso. A partir desse ponto, de posse dos valores numéricos obtidos, as tomadas de decisão são realizadas e o acionamento das saídas é feito de acordo com as regras ativas no instante considerado.

O controlador nebuloso embarcado em uma placa Arduino Mega foi então submetido a testes para averiguar a robustez dos sensores utilizados e para garantir a correta aferição dos valores de entrada das variáveis, bem como avaliar o processo de inferência e o acionamento das saídas. As Figuras 8 e 9 mostram a implementação do sistema proposto em um protótipo, com uma ventoinha, para a representação da variável de saída denominada ventilação, e um LED, para a representação da variável de saída denominada iluminação.

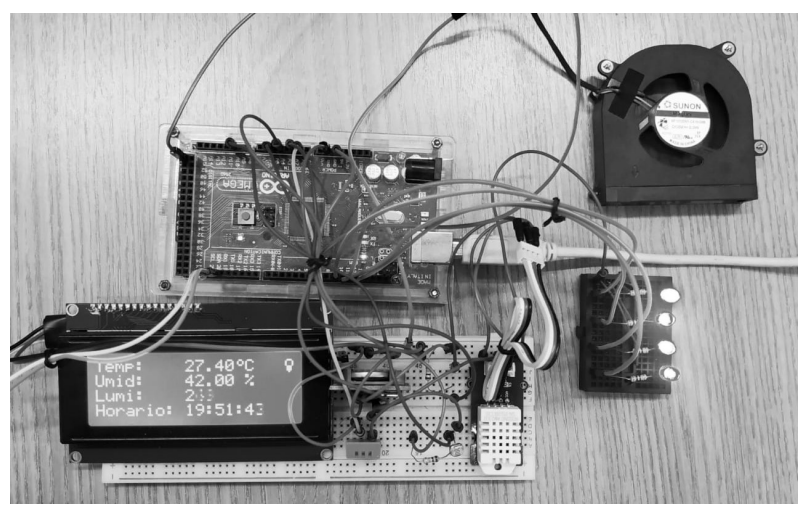

Figura 8. Detalhe da plataforma.

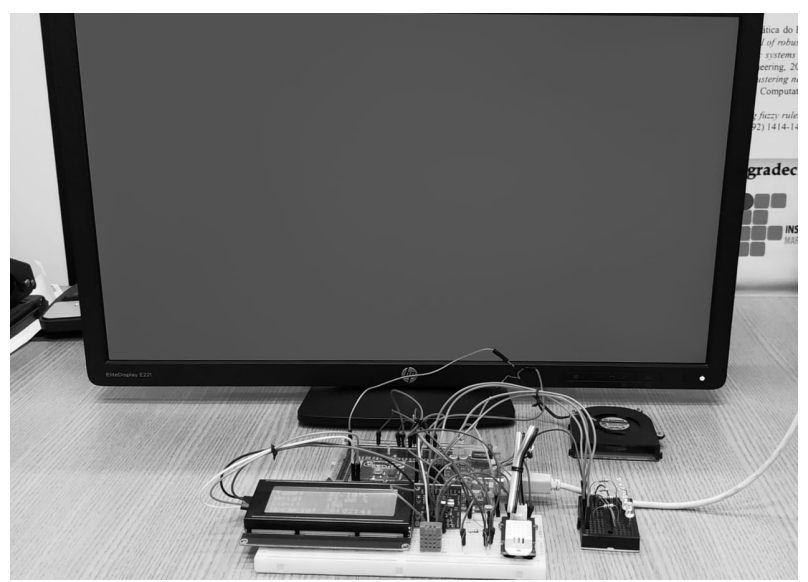

Figura 9. Protótipo implementado.

\section{RESULTADOS}

Os resultados experimentais mostraram a eficiência da metodologia proposta, uma vez que o sistema apresentou resultados satisfatórios para controle da temperatura e iluminação.
São mostradas as superfícies obtidas com a aplicação do método proposto, a partir de um modelo nebuloso Mamdani e método do centroide para as saídas ventilação e iluminação, nas Figura 10 e 11, respectivamente.

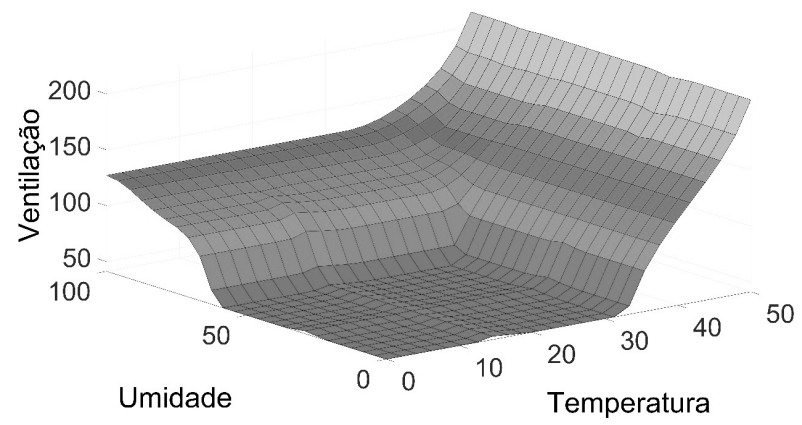

Figura 10. Superfície representando a ventilação em função da umidade e temperatura.

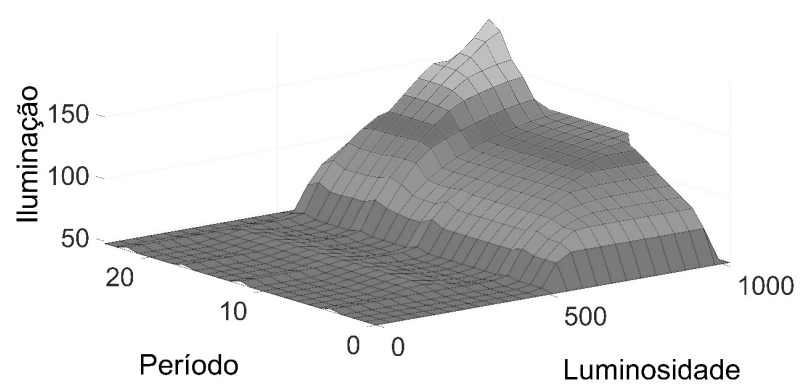

Figura 11. Superfície representando a iluminação em função do período e luminosidade.

\section{CONCLUSÕES}

A aplicação da lógica nebulosa se mostrou bastante adequada para o problema proposto. A variação suave das saídas - diferentemente dos sistemas com saídas $O N / O F F$ - permitiu um melhor gerenciamento do ponto de vista energético, uma vez que a potência fornecida à iluminação e à ventilação eram controladas conforme o horário e a luminosidade já presente no ambiente.

A facilidade de implementação do conhecimento do especialista e modificação da base de regra se mostra uma característica bem desejada, dado que possibilita que seja acrescentados instruções para controle do microclima de acordo com a cultura definida para cultivo. Assim, tornase possível cultivar vários tipos de hortaliças adaptadas a diferentes microclimas apenas inserindo novas regras.

Mais variáveis podem ser adicionadas, no entanto, o número de componentes necessários será maior, bem como o número de regras aumentará consideravelmente. Portanto, o sistema como um todo tornar-se-á bastante complexo. Contudo, como proposta futura, um modelo com mais variáveis será formulado. Ainda como proposta futura, tem-se a criação da interface sistema-usuário para exibição de dados e gerenciamento do sistema como um todo.

\section{AGRADECIMENTOS}

Os autores agradecem ao Instituto Federal do Maranhão - IFMA e à FAPEMA pelo apoio financeiro. 


\section{REFERÊNCIAS}

Alberoni, R.d.B. (1998). Hidroponia: como instalar e manejar o plantio de hortaliças dispensando o uso do solo. São Paulo: Nobel.

Almeida, C.C. (2014). Sistema eletrônico baseado em diodos emissores de luz (leds) para aplicação em estudos de fisiologia vegetal. Dissertação (Mestrado em Engenharia Elétrica) - Universidade Federal de Juiz de Fora.

Arduino (2017). analogread(). Disponível em: https : //www.arduino.cc/reference/en/language/functions/analogio/analogread/. Acessado em: 2018-03-20.

Azevedo Junior, A.L.d. (2016). Sistema de monitoramento e climatização de estufa de pequeno porte em um contexto doméstico.

Cavalcanti, J.H.F., de Melo, H., da Rocha Souto, C., Cavalcanti, M.T., and Rodrigues, M.C. (2012). Lógica fuzzy aplicada às engenharias. João Pessoa: Câmara.

de Abreu, C.S.P., Bastos, T.J., et al. (2015). Automação de abrigos de cultivo para culturas hidropônicas.

dos Santos, J.D., da Silva, A.L.L., da Luz Costa, J., Scheidt, G.N., Novak, A.C., Sydney, E.B., and Soccol, C.R. (2013). Development of a vinasse nutritive solution for hydroponics. Journal of environmental management, $114,8-12$.

Fonseca, F.A.d. and Schmitz, L. (2017). Sistema microcontrolado para estufas. B.S. thesis, Universidade Tecnológica Federal do Paraná.

Goldstein, H. (2018). The green promise of vertical farms [blueprints for a miracle]. IEEE Spectrum, 55(6), 50-55.

Hidroponia, T. (2013). Nft: Um tipo de hidroponia. Disponível em: http : //tudohidroponia.net/nft-umtipo - de - hidroponia/. Acessado em: 2018-03-14.

Hirzel, T. (2016). Arduino - pwm. Disponível em: https : //www.arduino.cc/en/Tutorial/PWM. Acessado em: 2018-03-20.

Jackson, S.D. (2009). Plant responses to photoperiod. New Phytologist, 181(3), 517-531.

JSM, L.M. and Sridevi, C. (2014). Design of efficient hydroponic nutrient solution control system using soft computing based solution grading. In Computation of Power, Energy, Information and Communication (ICCPEIC), 2014 International Conference on, 148-154. IEEE.

Lima, F.S. (2017). Sistema mimo inteligente nebuloso para controle de iluminação. XIII Simpósio Brasileiro de Automação Inteligente, Porto Alegre - RS, 858-863.

Loschiavo, R. (2015). O que é hidroponia? Disponvelem : http : //www.ecoeficientes.com.br/o - que - ehidroponia/. Acessado em: 2017-03-18.

Matsura, D.U., dos Santos, J.F., Maggieri, J.C., Nogueira, M., da Silva, L.F.R., and Silva, R.V. (2015). Sistema de controle de abastecimento de água e energia no cultivo hidropônico nft water supply control system and energy growing hydroponic nft.

Rebouças, P.M., Dias, Í.F., Alves, M.A., and Barbosa Filho, J.A.D. (2014). Radiação solar e temperatura do ar em ambiente protegido. Revista Agrogeoambiental, 7(2).

Rezende, S.O. (2003). Sistemas inteligentes: fundamentos e aplicações. Editora Manole Ltda.

Santos, P.d.S.d.M. (????). Horta hidropônica automatizada por microcontrolador.

Teruel, B.J. (2010). Controle automatizado de casas de vegetação: Variáveis climáticas e fertigação. Revista Bra- sileira de Engenharia Agricola e Ambiental-Agriambi, $14(3)$.

Zanette, A., Radanovitsck, E.A., and Gonçalves, W.W. (2013). Fuzzy logic (lógica difusa. Disponível em: http : //www.inf.ufrgs.br/ mrpritt/lib/exe/fet ch.php? media $=$ inf05508: $t-a-f u z z y . p d f$. Acessado em: 2017-03-14. 